\title{
ERRATUM TO: ON THE DIRAC COHOMOLOGY OF COMPLEX LIE GROUP REPRESENTATIONS
}

\author{
CHAO-PING DONG \\ School of Mathematics \\ Shandong University \\ Jinan 250100, China \\ chaoping@sdu.edu.cn
}

Abstract. We correct an error in the paper [D]. For Proposition 1.3 and Theorem 1.4 there to hold, we need to assume that $\lambda_{L}+s \lambda_{L}$ is dominant for $\Delta^{+}\left(\mathfrak{g}_{0}, \mathfrak{h}_{0}\right)$.

Let $G$ be a connected complex semisimple Lie group. Fix a Borel subgroup $B$ of $G$ containing $H$. Let us put $\Delta^{+}\left(\mathfrak{g}_{0}, \mathfrak{h}_{0}\right)=\Delta\left(\mathfrak{b}_{0}, \mathfrak{h}_{0}\right)$ and set

$$
\Delta^{+}(\mathfrak{g}, \mathfrak{h})=\Delta^{+}\left(\mathfrak{g}_{0}, \mathfrak{h}_{0}\right) \times\{0\} \cup\{0\} \times\left(-\Delta^{+}\left(\mathfrak{g}_{0}, \mathfrak{h}_{0}\right)\right)
$$

which is $\theta$-stable. As deduced by Barbasch and Pandžić [BP], to find all the irreducible unitary representations with non-zero Dirac cohomology, it suffices to consider $J\left(\lambda_{L},-s \lambda_{L}\right)$ such that $2 \lambda_{L}$ is dominant integral regular for $\Delta^{+}\left(\mathfrak{g}_{0}, \mathfrak{h}_{0}\right)$, where $s \in W$ is an involution. For Proposition 1.3 of [D] to hold, we need the additional requirement that $\lambda_{L}+s \lambda_{L}$ is dominant for $\Delta^{+}\left(\mathfrak{g}_{0}, \mathfrak{h}_{0}\right)$. Without this condition, that proposition can fail, as illustrated by the following example, told to me by Vogan.

Example. Take $G=S p(4, \mathbb{C})$, and identify $\mathfrak{h}_{0}^{*}$ with $\mathbb{C}^{2}$. Let us put

$$
\Delta^{+}\left(\mathfrak{g}_{0}, \mathfrak{h}_{0}\right)=\{(0,2),(1,-1),(1,1),(2,0)\} .
$$

Take $\lambda_{L}=(2,1)$. Let $s$ be the reflection in the root $(2,0)$. Then $\lambda_{R}=-s \lambda_{L}=$ $(2,-1)$. Let us consider the representation

$$
J((2,1),(2,-1))
$$

Now $\lambda_{L}-\lambda_{R}=(0,2)$, which is not dominant for $\Delta^{+}\left(\mathfrak{g}_{0}, \mathfrak{h}_{0}\right)$. Let $w$ be the reflection in the root $(1,-1)$. Then one can use the element $w \in W$ to conjugate $\lambda_{L}-\lambda_{R}$ to be the dominant one $\lambda:=\left\{\lambda_{L}-\lambda_{R}\right\}=(2,0)$, and use $\lambda$ to define a $\theta$-stable parabolic subalgebra $\mathfrak{q}=\mathfrak{l}+\mathfrak{u}$. But the point is that we should conjugate the Zhelobenko parameters $\lambda_{L}, \lambda_{R}$ simultaneously using the same $w \in W$, which then become

$$
J((1,2),(-1,2))
$$

DOI: $10.1007 /$ s00031-013-9226-9

Received March 4, 2013. Accepted March 25, 2013. Published online May 10, 2013. 
Now $2 w \lambda_{L}=(2,4)$, which has a negative pairing with the root $(1,-1)$ in $\mathfrak{u}$. Thus the above representation is not in the good range, and Proposition 1.3 of [D] fails: instead of being irreducible, the cohomologically induced module in the RHS of equation (8) of [D] now has five composition factors, one of which is $J((2,1),(2,-1))$.

Thus for Proposition 1.3 of [D] to hold, we need to assume that $\lambda_{L}+s \lambda_{L}$ is dominant for $\Delta^{+}\left(\mathfrak{g}_{0}, \mathfrak{h}_{0}\right)$. Accordingly, the correct version of Theorem 1.4 of [D] is stated as follows.

Theorem 1.4'. Let $J\left(\lambda_{L},-s \lambda_{L}\right)$ be an irreducible representation of $G$, where $2 \lambda_{L}$ is dominant integral regular for $\Delta^{+}\left(\mathfrak{g}_{0}, \mathfrak{h}_{0}\right)$, and $s \in W$ is an involution such that $\lambda:=\lambda_{L}+s \lambda_{L}$ is dominant for $\Delta^{+}\left(\mathfrak{g}_{0}, \mathfrak{h}_{0}\right)$. Let $\mathfrak{q}=\mathfrak{l}+\mathfrak{u}$ be the $\theta$-stable parabolic subalgebra of $\mathfrak{g}$ defined by $\lambda$. Then $J\left(\lambda_{L},-s \lambda_{L}\right)$ is a unitary $(\mathfrak{g}, K)$-module if and only if $J_{L}\left(\lambda_{L}^{\prime}, \lambda_{R}^{\prime}\right)$ is a unitary $(\mathfrak{l}, L \cap K)$-module. In such a case, if

$$
H_{D}\left(J_{L}\left(\lambda_{L}^{\prime}, \lambda_{R}^{\prime}\right)\right)=m 2^{\left[l_{0} / 2\right]} F_{2 \lambda_{L}-\rho(\mathfrak{u})-\rho_{c}^{L}},
$$

where $l_{0}=\operatorname{dim} \mathfrak{a}, m$ is a non-negative integer, and $F_{\nu}$ denotes the $\widetilde{K_{L}}$-type with highest weight $\nu$, then

$$
H_{D}\left(J\left(\lambda_{L},-s \lambda_{L}\right)\right)=m 2^{\left[l_{0} / 2\right]} E_{2 \lambda_{L}-\rho_{c}}
$$

where $E_{\mu}$ denotes the $\widetilde{K}$-type with highest weight $\mu$. In particular, $H_{D}\left(J\left(\lambda_{L},-s \lambda_{L}\right)\right)$ is non-zero if and only if $H_{D}\left(J_{L}\left(\lambda_{L}^{\prime}, \lambda_{R}^{\prime}\right)\right)$ is non-zero.

Since $2 \lambda_{L}$ is dominant integral regular, one sees easily that the additional assumption " $\lambda:=\lambda_{L}+s \lambda_{L}$ is dominant for $\Delta^{+}\left(\mathfrak{g}_{0}, \mathfrak{h}_{0}\right)$ " is equivalent to the requirement that $J\left(\lambda_{L},-s \lambda_{L}\right)$ is in the good range. Therefore, Theorem $1.4^{\prime}$ handles exactly the irreducible unitary representations of $G$ which are in the good range.

When $\lambda_{L}+s \lambda_{L}$ is not dominant, since we care the most about unitary representations, things can be remedied to a certain extent by adopting Parthasarathy's Dirac inequality, which requires that

$$
\left\|2 \lambda_{L}\right\| \leq\left\|\rho_{c}+\left\{\lambda_{L}+s \lambda_{L}\right\}\right\|
$$

For instance, the representation considered in the above example is not unitary. It is elementary to deduce the following: fix any $\lambda_{L}$ such that $2 \lambda_{L}$ is dominant integral regular, and any involution $s \in W$ such that $\lambda_{L}+s \lambda_{L}$ is not dominant; there exists a positive integer $N$ depending on $\lambda_{L}$ and $s$ such that

$$
\left\|2 k \lambda_{L}\right\|>\left\|\rho_{c}+\left\{k \lambda_{L}+s k \lambda_{L}\right\}\right\|,
$$

where $k \geq N$ is an arbitrary integer. Theorem $1.4^{\prime}$ cannot handle the possible unitary representations in the family $J\left(k \lambda_{L},-s k \lambda_{L}\right), 1 \leq k<N$.

Finally, we take this opportunity to correct the typos in [D]:

Page 72, line -4: replace "by $\Lambda^{+}$" with "by a subset of $\Lambda^{+}$".

Subsection 7.3: all the four places of $J\left(\lambda_{L},-\lambda_{L}\right)$ should be $J\left(\lambda_{L}, \lambda_{L}\right)$.

Acknowledgement. I thank Professor Vogan sincerely for pointing out the error to me. 


\section{References}

[BP] D. Barbasch, P. Pandžić, Dirac cohomology and unipotent representations of complex groups, in: Noncommutative Geometry and Global Analysis, Contemp. Math., Vol. 546, Amer. Math. Soc., 2011, pp. 1-22; also available at arXiv:1007.1289v1.

[D] C.-P. Dong, On the Dirac cohomology of complex Lie group representations, Transform. Groups 18 (2013), 61-79. 